\title{
An Adaptive Link Layer for Heterogeneous Multi-radio Mobile Sensor Networks
}

\author{
Jeremy Gummeson, Deepak Ganesan, Mark D. Corner and Prashant Shenoy
}

\begin{abstract}
An important challenge in mobile sensor networks is to enable energy-efficient communication over a diversity of distances while being robust to wireless effects caused by node mobility. In this paper, we argue that the pairing of two complementary radios with heterogeneous range characteristics enables greater range and interference diversity at lower energy cost than a single radio. We make three contributions towards the design of such multi-radio mobile sensor systems. First, we present the design of a novel reinforcement learning-based link layer algorithm that continually learns channel characteristics and dynamically decides when to switch between radios. Second, we describe a simple protocol that translates the benefits of the adaptive link layer into practice in an energy-efficient manner. Third, we present the design of Arthropod, a mote-class sensor platform that combines two such heterogneous radios (XE1205 and CC2420) and our implementation of the Q-learning based switching protocol in TinyOS 2.0. Using experiments conducted in a variety of urban and forested environments, we show that our system achieves up to $52 \%$ energy gains over a single radio system while handling node mobility. Our results also show that our system can handle short, medium and long-term wireless interference in such environments.
\end{abstract}

Index Terms-

\section{INTRODUCTION}

$\mathbf{M}$ OBILE sensor networks have received increased research attention recently with applications ranging from vehicular networks (e.g. DieselNet [2]) to animal tracking (e.g. ZebraNet [9]). The choice of the wireless radio is perhaps the single most crucial design parameter for designing a mobile sensor network. The wireless radio must enable node-to-node and node-to-basestation communication over distances dictated by application needs, while being energyefficient and robust to wireless effects introduced by mobility patterns. With advances in communication technologies, a spectrum of wireless radios are available to meet the needs of a sensor network. Table I depicts four common wireless radios used by today's sensor network platforms. As shown in the table, wireless radios are generally designed with a communication range in mind. For example, the Xtend and the XE1205 radios are designed for low-bitrate long-range communication over distances of a mile or more. In contrast, 802.11 and CC2420 radios enable high and low bandwidth communication, respectively, over short ranges of hundreds of feet or less. Thus, the sensor network designer must make

Manuscript received ; revised. This research was supported by NSF grants CNS-0626873, CNS-0615075, CNS-0520729, CNS-0546177, EEC-0313747, CNS-0916577, and CNS-0855128

The authors are with the Department of Computer Science, University of Massachusetts, Amherst, Amherst, MA 01003 (e-mail: \{gummeson, dganesan, mcorner, shenoy\}@cs.umass.edu).

Digital Object Identifier 10.1109/JSAC.2010.1009xx. a critical design choice. She can either choose a long-range radio enabling nodes to communicate over long distances but at the expense of expending more power. Or she can choose a shorter range radio that is more power-efficient but forego communication over longer distances.

Note that traditional techniques for range adaptation via power control or range elongation via the use of directional antennas do not address this tradeoff for mobile sensor networks. As shown in Table I, modern radios support range adaptation using power control - a higher power setting can be used to increase the communication range of the radio. While it is possible to choose a long range radio and use lower power settings for short range communication, doing so is far less efficient than using a short range radio for communicating over shorter distances. As shown in Table I, the lowest power setting on the XTend radio is still $561 \mathrm{x}$ more expensive than using the $\mathrm{CC} 2420$ radio. Using a radio at its maximum range is never desirable, as packet loss rates increase with distance; the radios mentioned in Table I typically have a packet loss rate of $\sim 30 \%$ at the reported distances, further emphasizing the need for appropriate radio hardware. Similarly, it is not feasible to use a radio designed for short range communication and to "increase" its range by using directional antennas. Directional antennas have been used successfully to increase the communication range of such radios - for example, the Mobisteer project [13]. However, since directional antennas are bulky, it is not feasible to deploy them in many mobile sensor network settings; for instance, animal tracking deployments require compact packaging of the mobile sensors.

In this paper, we pair two complementary radios with heterogeneous range characteristics to enable mobile sensor nodes the ability to achieve a significantly greater range diversity at a lower total energy cost when compared to a single radio. The use of multiple radios has been extensively investigated in the cellular community [28], but the radios employed in cellular devices are used to either maximize bandwidth or achieve interoperability. The key idea of our work is to operate each radio over a range where it is more energy efficient and to switch to the other radio whenever a mobile node moves from one radio's effective range to another. Specifically, we choose a high bandwidth spread spectrum radio with poor range characteristics and a variable bitrate radio tuned to have low bandwidth but better range. The two radios also operate in two isolated frequency bands - an impossibility with a single radio. In this manner, we achieve the best-of-both-worlds and eliminate the drawbacks of a single radio platform. An additional benefit of pairing 
TABLE I

A SPECTRUM OF RADIO HARDWARE

\begin{tabular}{|l|l|l|l|l|}
\hline Radio & Bandwidth & $\begin{array}{l}\text { transmit power levels } \\
(\min , \text { max }), \text { steps }\end{array}$ & $\begin{array}{l}\text { transmit energy/bit } \\
(\min , \mathrm{max})\end{array}$ & receive power \\
\hline \hline CC2420 & $250 \mathrm{Kbps}$ & $(-25,0 \mathrm{dBm}), 31$ & $102,208 \mathrm{~nJ} / \mathrm{bit}$ & $56.7 \mathrm{~mW}$ \\
\hline XE1205 & $38.1 \mathrm{Kbps}$ & $(0,15 \mathrm{dBm}), 4$ & $1803,5276 \mathrm{~nJ} / \mathrm{bit}$ & $42.0 \mathrm{~mW}$ \\
\hline XE1205 & $76.8 \mathrm{kbps}$ & $(0,15 \mathrm{dBm}), 4$ & $894,2617 \mathrm{~nJ} / \mathrm{bit}$ & $42.0 \mathrm{~mW}$ \\
\hline $802.11 \mathrm{~b}$ & $11 \mathrm{Mbps}$ &,$- 120 \mathrm{~nJ} / \mathrm{bit}$ & $80 \mathrm{~m}$ & $900 \mathrm{~mW}$ \\
\hline XTend & $9.6 \mathrm{kbps}$ & $57.3,380.2 \mathrm{uJ} / \mathrm{bit}$ & $240 \mathrm{~mW}$ & $80 \mathrm{~m}-800 \mathrm{~m}$ \\
\hline
\end{tabular}

complementary radios is that it enables adaptation to channel interference-by dynamically choosing the radio with the least interferences from other wireless devices. When using the two radios to adapt to interference, the variable bitrate radio is tuned for higher bandwidth but reduced range to better complement the alternate radio interface. The isolated frequency bands used by each radio allows robust adaptation.

We present the design of a heterogeneous multi-radio platform and system for handling range dynamics, where the choice of which radio to use for communication is made dynamically based on current channel characteristics, specifically wireless channel variations caused by device mobility and range effects. To shield applications from the increased complexity of choosing between radios, we present the design of a unified link layer that transparently chooses which radio to employ for communication between a pair of nodes. At the core of such a link layer is an adaptive algorithm that can dynamically decide when to use each radio for a wide range of mobility patterns. Such an algorithm is non-trivial since it needs to continually monitor and "learn" channel characteristics for the two radios and determine which one provides the lowest energy communication channel. Additionally, the practical implementation of such an adaptive link layer on sensor platforms presents a significant challenge since the energy and resource overhead for monitoring, learning, and switching between radios needs to be kept as low as possible.

\section{A. Contributions}

In this paper, we propose a multi-radio hardware and link layer solution for range-adaptive mobile wireless sensor networks. Our work has three major contributions:

Q-Learning based Unified Link Layer: Our first contribution is a reinforcement-learning based algorithm that enables adaptatation across radios with different power/range tradeoffs. This algorithm learns the characteristics of radio channels through exploration and continually adapts to use the more efficient one.

Multi-radio Switching Protocol: Our second contribution is a energy-efficient switching protocol that translates the benefits of the Q-learning based adaptation algorithm into practice. The protocol transparently switches between radios, thereby providing the abstraction of a unified link layer to applications executing on multi-radio platforms.

Heterogeneous Multi-Radio Sensor Platform: Our third contribution is the design of a new mote-class sensor platform, the Arthropod, that pairs two radios with complementary characteristics: the CC2420 and XE1205. These radios have very different maximum ranges ( 80 meters vs 800 meters), and also significantly differ in their maximum power output
( $0 \mathrm{dBm}$ vs $15 \mathrm{dBm}$ ) Thus, the Arthropod offers good potential for range adaptation to handle mobility effects.

We conduct mobility experiments using our hardware and software prototype in a variety of settings-urban/indoor, urban/outdoor, foliage - and for a range of mobility patterns - continuous and nomadic - that are typical in mobile sensor network deployments. Our experiments show that we obtain up to $52 \%$ improvements in energy efficiency over using only one of the two radios on the platform, while achieving a loss rate only marginally higher than using just the high-power radio. Our experiments on interference dynamics show that our link layer can adapt to short, medium and long-term wireless interference, while yielding a significant reduction in energy usage over a single radio system.

\section{RELATED WORK}

Since radio diversity presents clear benefits along a number of dimensions: energy, robustness to interference, increased bandwidth and ease of deployment, a number of multi-radio systems have been designed in recent years. This has primarily involved a separation of control tasks such as neighbor discovery or neighbor wakeup from data transmission. Such a separation has been achieved by pairing 802.11 with the CC2420 [12] or the CC1000 [10], [15], [23], 802.11 with a custom radio for Wake-On-Wireless [22], [18], and 802.11 with an XTend [26] radio [2] for the UMassDieselNet DTN [4]. While such static allocation of roles to radios offers useful benefits, it does not fully utilize the potential of multiradio systems. In our system, either radio can be used for control or data communication and the choice of which radio to use for communication is made dynamically based on current channel characteristics.

Multiple radio interfaces have also been exploited for increasing bandwidth and tolerating disconnection on mobile wireless devices. The Mobile Access Router [20] exploits multiple types of radio interfaces (eg. 802.11, GPRS, etc), or interfaces tied to different service providers to aggregate bandwidth and avoid stalled transfers. A related technique is PTCP that uses link-layer striping [8] to achieve a similar goal. All these mechanisms are aggressive in using multiple interfaces and do not take energy into account when choosing an interface. An updated Wake-On-Wireless system [1] and Context-for-Wireless [17] use 802.11 with cellular radios for data transmissions, with a static preference given to 802.11 when available.

One dynamic, energy-aware system is Coolspots, which combines 802.11 with Bluetooth [14]. Coolspots chooses Bluetooth transmission when available, and 802.11 when Bluetooth is insufficient to meet the bandwidth requirements. 
However, the choice of when to use a radio is made using coarse-grained feedback from the network layer, and neglects the benefits of a fine-grained, link-layer approach; this type of approach is useful because it allows a system to react quickly to short term dynamics. Another approach to dynamically utilizing a multi-radio system to achieve is found in [21] and [11]. These systems pair 802.11 with 802.15 .4 and chooses the appropriate interface based on data size; energy efficiency is achieved by batching packet transmission. Achieving efficiency by increasing latency is beneficial, but instead our work focuses on reducing energy consumption by reacting to variations in mobility and channel conditions. Other systems, such as Triage extend this paradigm from multiple radios to multiple platforms [3]; however in this work a single platform is sufficient to process data transmission from both radios.

Recent work on wireless mesh networks has explored designs with multiple radios per node. For instance, carefully planned mesh networks can exploit multiple radios to make channel assignment more effective [5]. However, these approaches have not addressed the problem of algorithms to dynamically react to changing channel characteristics, and do not consider energy efficiency.

In sum, it is our view that ours is the first system to use multiple low-power radios for link-layer, energy-aware, transmission of data, and constitutes an important step towards a fine-grained, energy-adaptive, multi-radio link layer.

\section{System Model AND Assumptions}

Our work assumes a dual-radio sensor platform where multiple heterogeneous wireless radios are available to a mobile sensor application. As noted earlier, multiple radios enable greater range diversity and also enable better adaptation to channel interference.

A possible downside to a multi-radio platform, however, is that it makes application design more complex. Since multiple radios are available, an application will need to decide which radio to use on a per-packet basis. To overcome this limitation, our work advocates transparency, where the presence of multiple radios is hidden from the application by a unified link layer. Such a link layer presents a "unified" logical radio to application and deals with the complexity of determining which radio to use for each outgoing packet. Applications use standard primitives to send messages as if they were using a single radio system, and leave it to the unified link layer to deal with the task of choosing a particular wireless interface for link layer communication with the next hop node.

The design of such a unified link layer raises three key questions: (i) what statistics should the link layer maintain for each wireless radio? (ii) Given these statistics and node mobility dynamics, what technique should it use to determine the best radio for communication at any instant? and (iii) What protocol should the sender and the receiver nodes use to switch to a different radio whenever the other radio becomes the better choice for communication?

The next three sections present our approach to deal with these questions. We first present a reinforcement learning approach, based on Q-learning, that uses simple link layer statistics to dynamically choose a radio. We then present a multi-radio switching protocol to allows a sender and a receiver to coordinate the switch from one radio to another whenever Q-learning determines such a switch is beneficial. We then present the hardware and software architecture of the Arthropod system that we have built to incorporate these mechanisms.

For the purposes of this paper and given our emphasis on mobile sensor networks, we will assume a multi-radio platform with a long-range XE1205 radio coupled with a shorter-range low-power CC2420 radio. However, the basic Qlearning framework and our switching protocol can be easily adapted to other radio combinations as well.

\section{Q-LEARning BASED MUlti-RAdio LinK LAYER}

In this section, we present a unified link layer that is driven by a learning algorithm that adapts radio state parameters based on packet statistics. To realize the decisions made by this algorithm, we also employ a radio-switching protocol that handles transitions between different hardware configurations. The result is a single-radio abstraction that is robust with respect to interference and mobility dynamics.

Mobile multi-radio systems regularly incur unpredictable and widely varying conditions due to channel effects such as shadowing, fading, and multi-path effects, as well as varying interference. While adapting to these dynamics, the channel has hidden state: conditions on the radio not being used. In order to avoid local minimum, the system must periodically attempt to explore other operating states.

In particular, we have chosen to use a reinforcement technique called Q-Learning that provides exactly the properties required: a simple reward for making correct decisions and an ability to explore other operating points periodically [24]. In this section, we introduce some concepts from reinforcement learning and outline the design of the adaptation algorithm that is at the core of our unified multi-radio link layer.

\section{A. Introduction to $Q$-Learning}

Q-Learning is a reinforcement-learning technique to enable decision-making for agents in an unknown environment [24]. An agent continually takes an action from a set of possible actions and observes some reward associated with the outcome of their decision. In Q-Learning, there is a "Q-Matrix" that updates according to the reward received, and the state transitions. This Q-Matrix is used to determine which action is optimum while an agent is in a given state; A Q-Matrix has a corresponding Reward-Matrix that contains the reward to be received by the agent for arriving at a particular state. In Q-Learning, the agent will occasionally take a random action to explore states that have not been visited for some time.

Algorithm 1 shows the procedure for Q-Learning [24]. The learning algorithm uses three parameters - the learning rate $\alpha$, discount factor $\gamma$, and the $\epsilon$-greedy parameter. The learning rate places a limit on how quickly learning occurs. If this parameter is set too low, it will take a long time for the system to learn, while if set too high, will cause the QValues to never converge to optimal values. The discount factor is used to determine how much emphasis is placed 


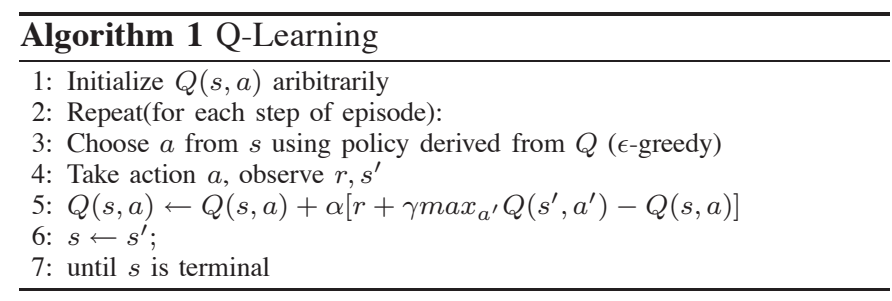

on future rewards. Setting this parameter low will optimize for immediate rewards, while setting this parameter high will place more importance on future rewards. Parameter $\epsilon$ determines with what probability a random action is explored, rather than choosing an action with the highest Q-Value.

\section{B. Designing a Unified Link Layer using Q-Learning}

We now describe how Q-Learning can be used to adapt between different radios in the case of a dual-radio sensor node. As noted earlier, we assume the availability of a multiradio platform with complementary radios (e.g. a short-range low-power and long-range high-power radio). In Section VI, we describe one such platform that we have designed that combines a CC2420 and XE1205 radio. We also assume a traffic model where mobile nodes periodically report sensor readings and transfer data between each other when in range.

We first consider the case where each radio is set to a single power level. In this case, Q-Learning uses a two state model (one for each radio) where the action taken by the agent is either to stay with the same radio or switch to the alternate radio. The agent will switch radios if conditions deteriorate on the current radio (or is disconnected), or if conditions improve on the alternate radio. These dynamics are captured in the Q-Matrix at a rate governed by $\alpha$ and $\gamma$ through feedback from packets transmitted over the current radio, as well as from exploration packets transmitted over the other radio (with frequency determined by $\epsilon$ ). The optimal rate exploration packets should be sent is determined largely by mobility rate and interference dynamics; ideally this rate would be determined adaptively. If the agent finds that the alternate radio has a higher Q-Value (i.e. lower energy consumption), the agent will choose to use this new radio interface.

This two state model may be expanded to an $n$-state model, where each state represents a radio at a particular transmit power level, each representing a particular range/power tradeoff. For example, four states would be required for two radios, each with two transmit power level options. However, increasing the number of states comes at the cost of either increased exploration overhead or decreased exploration frequency since exploration requires time and energy. We reduce this overhead in the $n$-state case by considering only three states at a time the current state and two adjacent states, a lower-range/lowerpower state, and a higher-power/higher-range state. Both these adjacent states could be on the same radio or a different radio. Thus, exploration is limited to only two states at any time.

Reward matrix: A key aspect of Q-Learning adaptation is defining the reward matrix $R$ for each state. The unified link layer receives information about the number of retransmission attempts and number of congestion backoffs for each packet that it transmits through either radio; these metrics are used to determine the reward for the current choice of radio / power level. We model the reward as an estimate of the amount of energy associated with the channel metrics collected for a given packet. The amount of energy to transmit a given packet is a function of packet size, static radio parameters such as receive/transmit power and channel sense time, number of retransmission attempts, and the number of congestion backoffs. Energy is a cost, rather than a reward, so its value is negative. The following equation shows how rewards are calculated where $i$ is the number of retransmissions:

$$
\begin{aligned}
r[i]=\quad & (i \cdot(\text { PacketSize } \cdot \text { ByteTime } \cdot \text { TxPower }+ \\
& \text { AckTimeOut } \cdot \text { RxPower }) \\
+ & \text { RxPower } \cdot(\text { AckRTT }) \\
+ & \text { PacketSize } \cdot \text { ByteTime } \cdot \text { TxPower })
\end{aligned}
$$

While the above equation determines the reward when a packet is successfully transmitted, we also need to consider the case when a packet is unsuccessful after a pre-defined maximum number of retries. In this case, we want the QLearning algorithm to progressively try higher power states until it reaches the highest power state. To obtain this behavior, when a packet transmission is unsuccessful on a low-power state, we assign a large negative reward to encourage the algorithm to switch to a higher power state sooner, thereby limiting the number of lost packets. We achieve this behavior by selecting a policy for choosing an action, $a$, from the set of actions, $s$, with the maximal $\mathrm{Q}$ value or the minimal expected energy consumption for a packet transmission. Once the highest power state is reached, if packet transmission is still unsuccessful, a zero reward is assigned since there is no point in switching back to other lower power states until the connection is re-established at the high-power state.

\section{Multi-Radio Switching Protocol}

Translating the Q-Learning based switching algorithm to a working protocol presents a non-trivial challenge. When a sender decides to switch to or explore another radio, it needs to notify the receiver of such an action. However, an explicit handoff may not always work, for example, the receiver may be unreachable by the current radio due to mobility. A trivial solution would be for the receiver to keep both radios always active, obviating the need for handoff. However, this option is clearly inefficient as it requires both radios to be in receive mode, consuming significant energy. Thus, a key challenge that we address is: how can we design a practical protocol for switching between radios that is energy-efficient and reliable?

In the rest of this section, we describe the sender and receiver side design for our adaptive multi-radio block transfer protocol. For simplicity, we consider a dual-radio system with a high-power radio (HIGH) and low-power radio (LOW) with only one power level per radio.

\section{A. Sender State Machine}

The state machine at the sender is shown in Figure 1. We first describe the normal operation of the state machine before discussing how we handle exceptional cases that arise due 


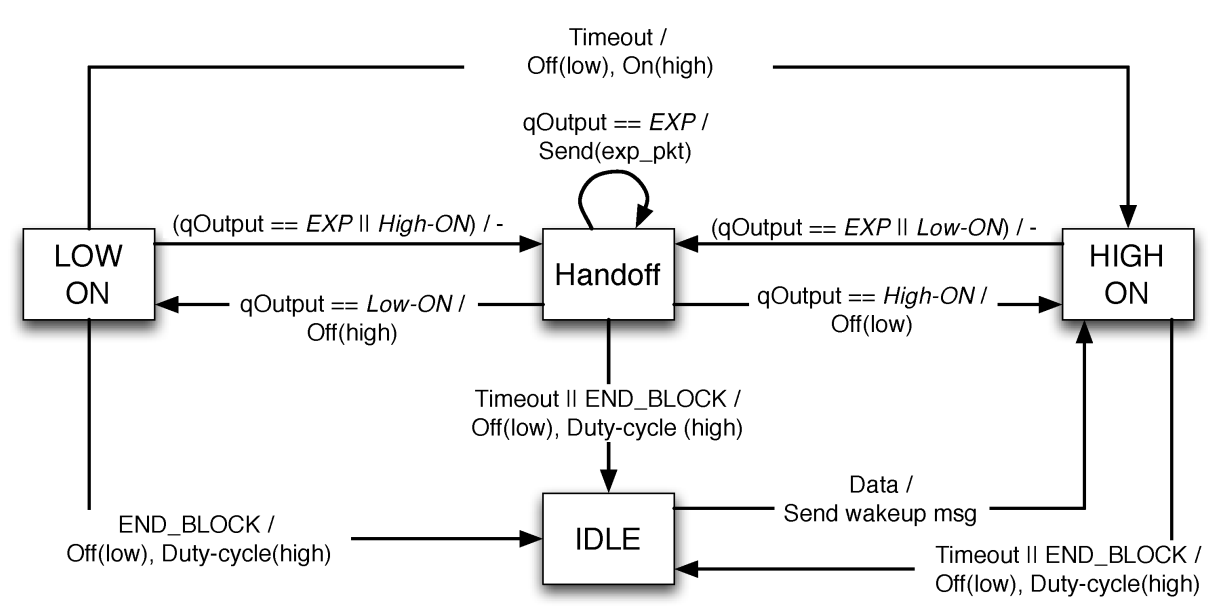

Fig. 1. Sender state machine. qOutput denotes the output of the Q-Learning algorithm, which can be either explore, turn on low-power radio (low), or turn on the high-power radio (high). Transitioning from the IDLE state requires a wakeup message.

to losses and disconnections. When data transfer starts, the sender first needs to "wakeup" the receiver from its IDLE state. There are many approaches to duty-cycling and wake up (e.g. SMAC [27], BMAC [16]), and we assume that one of these approaches are available for the radio. Once the wakeup command is successful, the sender transitions from IDLE to HIGH-ON state.

Switching and exploration between the radios requires a handshake between the sender and receiver; first, the sender sends a packet indicating that a switch needs to be done, and if the packet is transmitted successfully, the sender and receiver can synchronously switch states to the second radio or explore on it. To perform such a handshake, the sender state machine includes a handoff state in which both radios are turned on. To illustrate, consider a switch from the HIGHON to LOW-ON state triggered by the Q-Learning algorithm. The state machine first sends a data packet while remaining in the current state with the handoff flag set. If the packet is successfully transmitted, the state machine transitions to the HANDOFF state. (Note that the receiver is in the BOTHON state at this point and can receive on both radios). From this state, the sender can send a packet on the LOW radio to transition to LOW-ON state. A similar process is done during exploration. The sender and receiver transition synchronously to the HANDOFF and BOTH-ON states respectively, and stay in this state until exploration is complete, after which they switch back to whatever state they were in earlier.

Finally, we also deal with various cases where the state machines at the sender and receiver may become out-of-sync due to lost packets/acks, or complete loss of connectivity on one or both radios. If the LOW radio is currently in use and becomes disconnected, the sender times out, transitions to the HIGH-ON state and attempts to transmit using the long range radio. (Note that the receiver switches to the BOTH-ON state after a similar timeout, and is ready to receive on the HIGH radio.). If this fails as well, then after another timeout, the sender switches to IDLE mode since it means that the sender and receiver are out-of-range of both radios.

\section{B. Receiver State Machine}

The state machine at the receiver is shown in Figure 2. When data transfer starts, the receiver is in the IDLE state, where it operates with the HIGH radio in duty-cycled mode, and the LOW radio in off mode. This enables wakeup by the long-range radio to maximize contact time between the sender and receiver. The receiver is woken up out of this state by a long preamble on the HIGH radio, and switches to the $H I G H$ $O N$ state. Switching between the two radios occurs through a handoff state where both radios are switched on and ready to receive. When the receiver gets a packet with the handoff flag set, it transitions to the BOTH-ON state. It stays in this state until the sender informs the receiver to switch to either the LOW-ON or the HIGH-ON state. The receiver transitions back to the IDLE mode when the END_BLOCK flag is set in a packet indicating that the sender has completed the current transfer of a block.

The receiver state machine also handles a number of exceptional cases that may arise. When the receiver is in the LOW-ON or HIGH-ON state and does not receive a packet for a short duration, it transitions to the BOTH-ON state. This enables the receiver to deal with two cases: (a) the sender is using one radio whereas the receiver is out-of-sync and listening on the other radio, (b) the sender is out of range of the current radio but in range of the other radio. If no packet is received in the BOTH-ON state, it implies that the sender has dropped out of contact of both radios, therefore the receiver switches back to the IDLE state.

Summary of benefits: Having described the sender and receiver state machines, we now briefly describe the main benefits of our switching protocol.

- Active mode efficiency: During a block transfer, we minimize the amount of time for which both radios are turned on at the sender and receiver. This ensures that our system almost always consumes only as much energy as a single radio system.

- Low packet overhead: All state transitions in our protocol are triggered by flags set in data packets. There are no additional control packets, hence our protocol has extremely low packet overhead. 


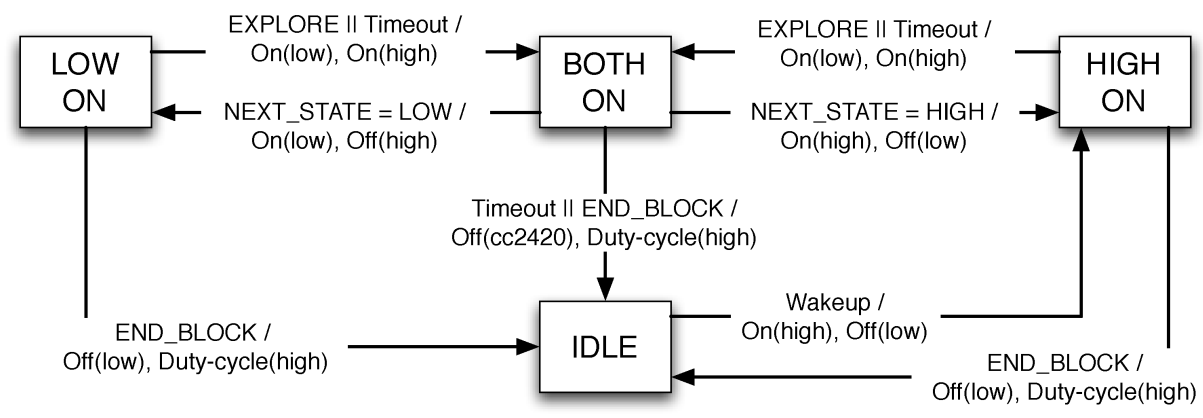

Fig. 2. Receiver state machine

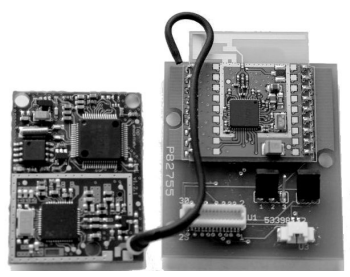

(a) Hardware Prototype

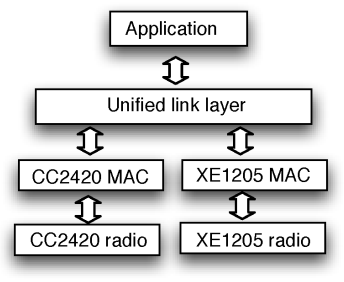

(b) Software Architecture
Fig. 3. System Components: (a)Hardware prototype comprising the Tinynode and a CC2420 expansion board and (b)Unified Link Layer for the radios

- Robustness: Our protocol is robust to channel vagaries and different mobility patterns, and can recover from lost packets/acks, disconnections, and out-of-sync states.

\section{ARTHROPOD IMPLEMENTATION}

We have built a prototype multi-radio platform called Arthropod and have implemented the Q-Learning based adaptive link-layer and switching protocol. This section describes the hardware and software implementation of our system.

\section{A. Hardware Architecture}

Our Arthropod sensor platform consists of a low-power microcontroller and a pair of heterogeneous low-power radios. The current prototype employs a MSP430 microcontroller, a CC2420 radio, and an XE1205 radio. Rather than constructing such a platform from scratch, we employed an existing Tinynode sensor platform [6], which contains a MSP430 processor and the XE1205 radio, and augmented it with a custom-built daughterboard comprising the CC2420 radio. We constructed the daughterboard by connecting an EasyBee CC2420 evaluation board [19] to several GPIO pins and an SPI bus available on the Tinynode. Figure 3(a) depicts the resulting prototype hardware of Arthropod.

The particular choice of the XE1205 and the CC2420 radios was governed by their complementary characteristics (see Table I). The two radios operating in mutually exclusive frequency bands $-900 \mathrm{MHz}$ and the $2.4 \mathrm{GHz}$ for the XE1205 and CC2420, respectively - enabling better interference adaptation. The table also shows that when operating at $0 \mathrm{dBm}$, both radios yield a range of $80 \mathrm{~m}$. However, the energy figures also indicate that if 5 or more retransmissions are needed on the CC2420, it is cheaper to use the XE1205 instead. In practice, retransmissions are more expensive since the sender needs to keep the radio active to receive the acknowledgment.

Arthropod also enables range diversity. While the peak range of the XE1205 is $2 \mathrm{kms}$ for a bandwidth setting of $1.2 \mathrm{Kbps}$ and $+15 \mathrm{dBm}$ power level, we were unable to get reliable transmission on the XE1205 at this setting due to known calibration problems with the TinyNode's XE1205 radio. Therefore, we use a data rate of 38.1 Kbps @ $+15 \mathrm{dBm}$, at which setting the maximum range is $800 \mathrm{~m}$. In contrast, the CC2420 cannot transmit beyond $0 \mathrm{dBm}$ and thus has a maximum range of $80 \mathrm{~m}$.

\section{B. Software Architecture}

The software implementation for Arthropod is an adaptive link-layer that unifies the individual MAC layers for the two radios. We have implemented a unified radio interface as part of the TinyOS-2.x operating system for motes [25]. The unified radio interface consists of two primary components: TinyOS-2.x drivers for the XE1205 and CC2420 radios and a unified link layer that manages the radio drivers. Figure 3(b) shows the arrangement of these software components. More implementation details may be found in [7].

\section{EXPERIMENTAL EVALUATION}

In this section, we present a detailed evaluation of the Q-learning based unified link layer using a combination of experiments using data traces, results from Q-Learning running on an Arthropod mote, as well as implementation benchmarks. Our evaluation has four parts. First, we evaluate the performance of the Q-learning link layer in adapting to a diverse set of mobility patterns. Second, using traces we evaluate how well the learning algorithm handles power control across the two radio interfaces. Third, we evaluate the efficacy of Q-learning for handling interference dynamics. Finally, we present benchmarks from an implementation of the link layer for an Arthropod mote to demonstrate that the described Q-Learning algorithm is efficient and has low resource usage.

\section{A. Datasets}

To ensure repeatable experimentation of the link layer, we gathered datasets under different conditions using our 
TABLE II

BRIEF SUMMARY OF MOBILITY TRACES

\begin{tabular}{|c|c|l|}
\hline Environment & Mobility Pattern & example scenario \\
\hline \hline urban-indoor & continuous w/ obstructions & people in a building \\
\hline urban-outdoor & continuous partial LOS & moving vehicle \\
\hline urban-outdoor & nomadic & bus w/ stops \\
\hline foliage & nomdic & bus w/ stops \\
\hline
\end{tabular}

TABLE III

SUMMARY OF INTERFERENCE TRACES.

\begin{tabular}{|c|c|l|}
\hline Dataset & Interference Type & Description \\
\hline \hline Long Interference & 30 minute periods & $\begin{array}{l}\text { indoors; 100ft range; } \\
\text { XE1205@0dBm }\end{array}$ \\
\hline Medium Interference & $200-235$ packet bursts & $\begin{array}{l}\text { indoors; 100ft range; } \\
\text { XE1205@00dBm }\end{array}$ \\
\hline Short Interference & $25-32$ packet bursts & $\begin{array}{l}\text { indoors; 100ft range; } \\
\text { XE1205@0dBm }\end{array}$ \\
\hline Low Interference & ambient & $\begin{array}{l}\text { indoors;100ft range; } \\
\text { XE1205@0dBm }\end{array}$ \\
\hline
\end{tabular}

hardware prototype. We obtained four types of datasets that are a good representation of mobility patterns found in mobile sensor network deployments. Two of these represent nomadic movement patterns in urban and rural settings. The other mobility traces gathered represent continuous mobility both in indoor and outdoor environments. A summary of these datasets is presented in Table II. We then gathered four additional datasets that represent different types of interference dynamics. Three of these datasets are created by introducing interference bursts of varying lengths from other sensor motes. The other dataset consists of ambient interference sources found in typical office buildings (e.g. 802.11, bluetooth). These datasets are briefly desribed in Table III.

The datasets were obtained from two Arthropod motes one Arthropod mote sends 20 byte packets with increasing sequence numbers over both radio interfaces at a fixed rate (2 Packets / second). A second mote places both radios in receive mode and acknowledges all packets received on each radio interface. For each packet, the sending node records the number of congestion backoffs experienced while trying to send the packet, as well as the number of retransmissions before receiving an acknowledgement from the receiver. The maximum number of backoffs is set to 6 , and retransmissions is set to a limit of 10 , after which the link layer at the sender gives up on the transmission.

In addition to link layer statistics we also store the radio chosen by the Q-learning algorithm for each packet. This allows us to verify that the algorithm is functioning correctly by comparing the decisions to the losses seen on each interface. For the mobility datasets, we configure the long-range XE1205 radio to a data-rate of $38.1 \mathrm{kbps}$ at $15 \mathrm{dbm}$, whereas the shortrange CC2420 radio uses a data-rate of $250 \mathrm{kbps}$ at $0 \mathrm{dbm}$.

\section{B. Evaluation of Q-Learning for Mobility Dynamics}

These experiments evaluate the performance of the Qlearning algorithm in a MATLAB simulation environment and its performance for the various mobility traces described above. To get an accurate measure of the performance of the Q-learning based link layer, we emulate the behavior of the sender and receiver state machine (Section IV) given the sequence of packet losses observed in the traces. (Later, in

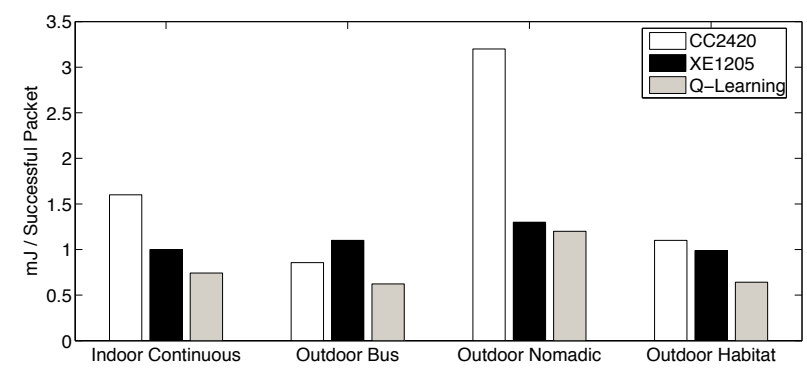

Fig. 4. Energy consumed per successful packet for each dataset and strategy

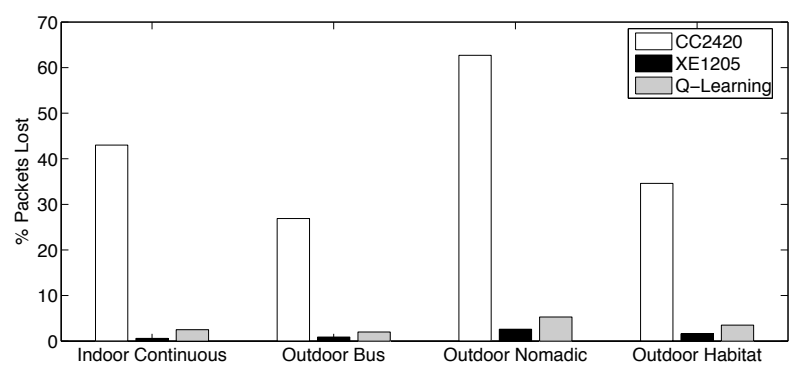

Fig. 5. Percent Packets lost for the two radio interfaces and Q-Learning implementation

Section VII-E we show that this emulation accurately corresponds to the performance of the real protocol in practice.)

For all datasets, we used an identical set of Q-Learning parameters: $\alpha=1.0, \gamma=0.7$ and $\epsilon=0.025$. These parameters were chosen since they seem to work well across a range of mobility datasets.

Q-Learning Performance: Figure 4 and Figure 5 summarize the energy per successful packet transmission and loss rates observed by our adaptive multi-radio link layer in comparison with using just one of the radios. In terms of energy consumption, the Q-Learning approach reduces energy consumption compared to the XE1205 radio by an average of $27 \%$ (the maximum reduction is $53.6 \%$ for the Outdoor Bus dataset), while incurring roughly $2-4 \%$ increased loss across the four cases. The slightly increased loss rate of Q-learning is caused by exploring an alternate interface periodically and transient losses caused while the algorithm is still learning. Similar energy gains of a maximum of $62.5 \%$ and an average of $44.6 \%$ are obtained over an approach that just uses the CC2420 radio but the improvements in loss rate are significantly higher $(25 \%-60 \%)$. The results show that in all cases, the energy consumption of the adaptive multi-radio link layer is better than exclusively using either the CC2420 or XE1205 radio, while keeping the link loss rate to be close to that observed by the long-range XE1205 radio.

As can be seen, the worst case for the Q-Learning protocol is the outdoor nomadic trace where our benefits are only marginal in terms of energy. This is because connectivity using the CC2420 radio is highly sporadic and also very lossy (65\% loss). Thus, our link layer is unable to take advantage of the $\mathrm{CC} 2420$ radio due to the high dynamics on it.

In summary, our results show that Q-learning can provide significant gains in terms of energy while only increasing packet loss marginally; when an opportunity arises for com- 
TABLE IV

Statistics for Dual Radio / Power Control Trace

\begin{tabular}{|c|c|c|}
\hline radio/power-level & \% packets lost & energy consumed \\
\hline \hline XE1205@0dBm & 4.24 & $.659 \mathrm{~mJ} / \mathrm{Tx}$ Success \\
\hline XE1205@15dBm & 0 & $.925 \mathrm{~mJ} / \mathrm{Tx}$ Success \\
\hline CC2420@-25dBm & 37.01 & $1.1 \mathrm{~mJ} / \mathrm{Tx}$ Success \\
\hline CC2420@0dBm & 35.45 & $1.2 \mathrm{~mJ} / \mathrm{Tx}$ Success \\
\hline Q-Learning & 3.53 & $.430 \mathrm{~mJ} / \mathrm{Tx}$ Success \\
\hline
\end{tabular}

munication over the CC2420 radio interface, our unified link layer is capable of exploiting its increased energy efficiency.

\section{Algorithm performance for power control across radios}

A logical extension to the unified link layer is handling transmission power control in addition to radio selection. The CC2420 radio is capable of transmitting packets from $-25 \mathrm{dBm}$ up to $0 \mathrm{dBm}$, while the XE1205 can transmit from $0 \mathrm{dBm}$ to $15 \mathrm{dBm}$. Increasing transmit power will provide longer range connectivity but uses additional energy; the optimum strategy will choose the minimum transmit power level on the most efficient radio without significantly increasing loss rate.

To evaluate power control across radio interfaces, we collected a packet trace similar to the Indoor Continuous described earlier. In addition to logging retransmissions and backoffs for the XE1205@15dBm and CC2420@0dBm,we log similar statistics for the XE1205 and CC2420@0dBm and $-25 \mathrm{dBm}$ respectively. The number of states in the Q-Learning algorithm increases from 2 to 4 ; we maintain a Q-value for each radio/power level combination. To reduce exploration overhead, we only explore the radio/power combinations adjacent to the current setting. Logically, the next setting expected from a mobile node would be one higher if the distance between sender and receiver has increased and one lower if the distance has decreased. Such an approach would scale even if there were more power states being considered per radio.

Table IV summarizes the results and compares the Qlearning approach to just using one of the two radios at one of the power levels. As can be seen, Q-learning is $54 \%$ better in terms of energy consumption per successful transmission than only using the XE1205 radio at $15 \mathrm{dBm}$ but has comparable loss rate. The energy benefits over using the $\mathrm{CC} 2420$ radio are $64 \%$; the loss rate also reduces by an order of magnitude. Overall, the Q-Learning based adaptive algorithm sends roughly $40 \%$ and $10 \%$ of the packets using $\mathrm{XE} 1205$ at $0 \mathrm{dBm}$ and $15 \mathrm{dBm}$; and $25 \%$ of the packets on the $\mathrm{CC} 2520$ at $-25 \mathrm{dBm}$ and $0 \mathrm{dBm}$. The results validate that QLearning is able to utilize each power state opportunistically.

These results show that the unified link layer is very effective at handling power control across multiple radio interfaces. Our scheme uses $2.5 x$ less power than the highest power radio with only a negligable packet loss increase. Each setting is used effectively at the appropriate range.

\section{Evaluation of Q-Learning for Interference Dynamics}

Our next set of experiments evaluate the performance of the Q-learning algorithm and its adaptability to changing interference conditions. Like before, these experiments were performed in MATLAB, using the data traces described in the previous section as input.

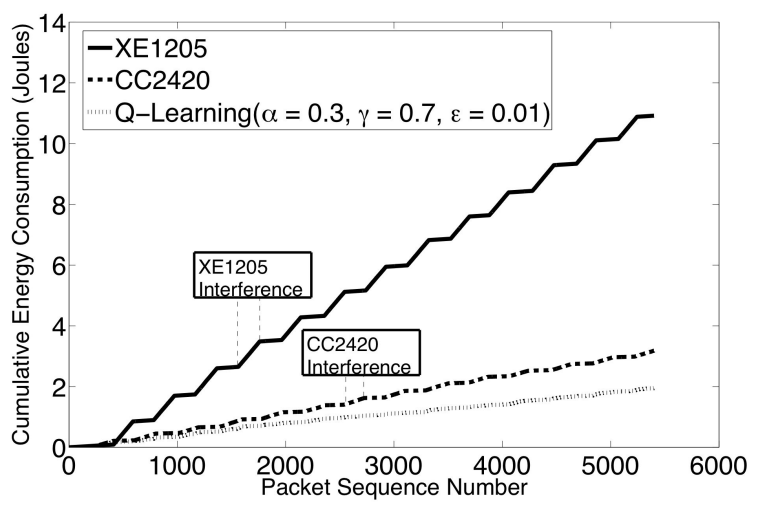

Fig. 6. Cumulative energy consumption for Long Bursts of Interference

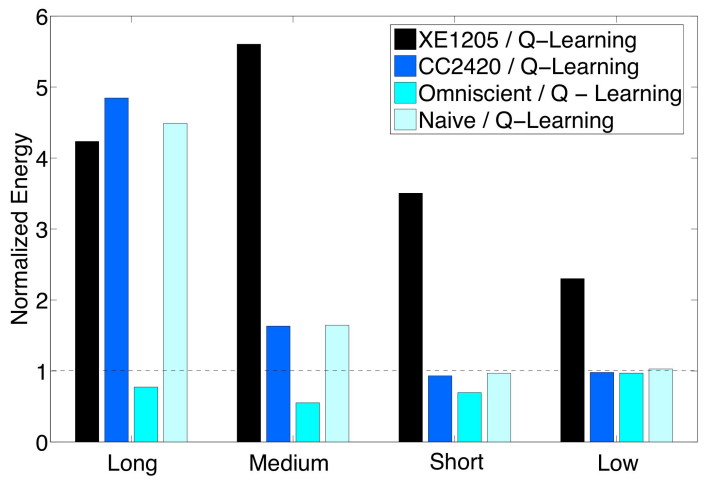

Fig. 7. Relative performance of Q-Learning for different interference patterns: (1) Long, (2) medium, (3) short, (4) low external interference

We compare the Q-learning algorithm against two alternate techniques. As a baseline, we use an omniscient strategy that has knowledge of the complete dataset, and is always able to make an optimal decision regarding which radio should be used. It is important to note that it is impossible for this strategy to be realized in practice - it is only used as a baseline to gauge how closely Q-Learning performs relative to such a best case strategy. The second is a non-adaptive naïve approach that only looks at the first 100 packets sent across each radio interface. This approach takes the ratio of cumulative energy consumption expended by each radio for this set of packets, and determines a probability $p$, corresponding to the the ratio of energy expended by the CC2420 vs XE1205 radio. For all other packets, the link layer transmits the packet on the $\mathrm{CC} 2420$ radio with probability $p$, and on the XE1205 radio with probability $1-p$.

Temporal Adaptability: First, we illustrate the adaptability of Q-Learning using a time-series plot of the medium interference trace, shown in Figure 6. The staircase shape of the CC2420 and XE1205 radios cumulative energy consumption is caused by bursts of interference introduced by the Telos motes and Tinynodes. The portions of the plot with a steep slope indicate that the radio is consuming an increased amount of energy per packet because of congestion and interference. Every time interference is encountered, this causes an entry in the Q-Matrix to grow increasingly negative at a rate determined by the reward $r$ (energy consumption) 
TABLE V

Time Spent During Different Receive States

\begin{tabular}{|c|c|}
\hline Switching Protocol State & \% Time Spent \\
\hline \hline HIGH ON & 10.7 \\
\hline LOW ON & 78.1 \\
\hline BOTH ON & 11.2 \\
\hline
\end{tabular}

and Q-Learning parameters $\alpha$ and $\gamma$. Q-Learning settles on the radio with best channel characteristics, and periodically explores the other radio $(\epsilon=0.01)$. This gives Q-Learning the opportunity to adapt when communication patterns change in the network. As a result, the Q-Learning plot represents a hybrid of the CC2420 and XE1205 energy plots, where the radio with a minimum slope is chosen during each burst after a brief learning period, resulting in a smoothing of energy consumption over time with consistently better performance than each individual radio.

Aggregate Q-Learning Performance: Figure 7 summarizes how Q-learning performs relative to other schemes across all the datasets. For both long and medium term interference, Q-learning performs extremely well compared to choosing a single radio or the multi-radio naïve scheme. For longterm interference, the XE1205 radio consumes 4.2 times more energy than our system, the CC2420 consumes 4.8 times more energy, and the naïve algorithm consumes 4.5 times more energy. For medium time-scale interference, the XE1205 radio alone consumes 5.6 times more energy than is consumed by our algorithm, and the $\mathrm{CC} 2420$ radio consumes roughly 1.6 times more energy, while the naïve two-radio algorithm also uses around 1.6 times more energy. The gains are intuitive since the naïve algorithm is non-adaptive and assumes that the behavior across the first 100 packets will be representative of future channel conditions. The Q-learning algorithm, however, is not as efficient as the omniscient approach which consumes $77 \%$ of the energy used by Q-Learning in the case of long-term interference and $69 \%$ of the energy used by Q-Learning in the case of short-term interference. This is because the omniscient algorithm wastes no energy exploring the two channels and also is never impacted by channel dynamics.

The short interference trace represents a case where Qlearning can be expected to perform badly since the channel is switching behavior every 30 packets. When we choose the exploration factor, $\epsilon$, to be small, it is difficult to learn with few samples that the current radio-channel has become poor and the other radio channel has improved from its previous state. When undersampling the channel in this manner, the learning algorithm eventually converges on following the radio that uses less overall energy, but does not make improvements beyond the better of the two radios. The low interference trace represents a scenario where the environment has limited dynamics since there is very little external interference. For this scenario, our Q-Learning algorithm will choose the more energy-efficient radio (CC2420) and encounter a slight amount of overhead as a result of exploring the more energy-expensive channel periodically. In both these cases, Q-learning is almost as efficient as choosing the best radio.

In summary, our results show that $Q$-learning can provide significant performance gains when there is medium and

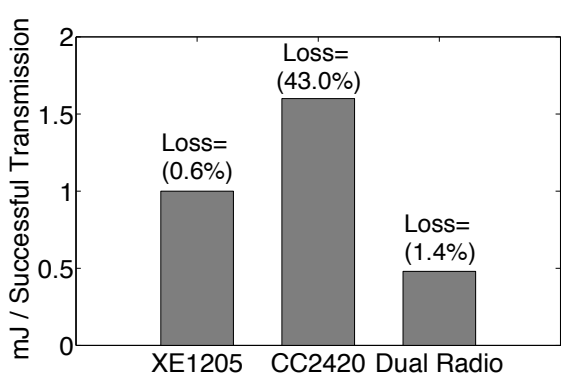

(a) Sender

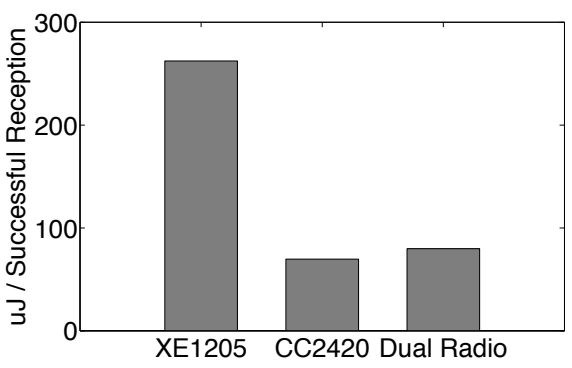

(b) Receiver

Fig. 8. Energy spent per packet by the sender and receiver. Labels on sender bars indicate packet loss rates.

long term interference that is greater than 200 packets in length. Even in hard to learn conditions such as short bursts of interference, and low interference conditions with limited dynamics, $Q$-Learning performs only marginally worse than the better of the two radios.

\section{E. Implementation Results}

To validate our implementation and show the performance of the radio switching protocol, we collect a new dataset with the same mobility pattern as the indoor continuous dataset. For this experiment, a pair of sender and receiver nodes run the switching protocol and Q-Learning algorithm online. This study aims to measure the actual per packet energy costs incurred by the sender and receiver. In particular, the receiver can become out-of-sync with the sender, resulting in the receiver turning both radios on, or timing out, all of which costs energy and results in more packet losses. For the mobility rates used in our experiments we chose a timeout value of 2 seconds. Finally, we also breakdown the $\%$ time spent by the receiver in different states of the receiver state machine.

The maximum data rate achievable by our software implementation for a pair of nodes transmitting continuously is $70 \mathrm{kbps}$. However, while logging packet statistics to the external flash, the data rate reduces to $14 \mathrm{kbps}$. This lower data rate is a result of the Tinynode platform multiplexing the SPI bus between the XE1205 radio and the external flash memory. As a result, the sender and receiver are not continuously sending data which causes idle gaps to appear between packets. This forces the receiver to expend additional energy while waiting for packets to arrive. Since the idle time is an artifact of our evaluation, we ignore these periods when presenting results.

To understand the energy efficiency of our protocol at the sender, Figure 8(a) compares the energy consumed by a single 
TABLE VI

LATENCY AND ENERGY CONSUMPTION FOR LINK-LAYER COMPONENTS

\begin{tabular}{|l|c|c|}
\hline Task & Latency & Energy \\
\hline \hline Radio Selection & $19.3 \mathrm{us}$ & $104 \mathrm{~nJ}$ \\
\hline Q-Matrix Update & $43.6 \mathrm{us}$ & $235 \mathrm{~nJ}$ \\
\hline $\begin{array}{l}\text { Transmit 20-Byte packet (XE1205 @ 0dBm } \\
\text { and 76.8kbps) }\end{array}$ & $3.9 \mathrm{~ms}$ & $218 \mathrm{uJ}$ \\
\hline Transmit 20-Byte packet (CC2420 @ 0dBm) & $1.7 \mathrm{~ms}$ & $92 \mathrm{uJ}$ \\
\hline
\end{tabular}

radio strategy to that of the dual radio implementation. The per packet energy consumption numbers presented for the CC2420 and XE1205 only cases are from Section VII-B. The results in Figure 8(a) show that our adaptive algorithm is $64 \%$ more efficient than a CC2420-only scheme, and $43 \%$ more efficient than an XE1205-only scheme verifying the gains found in simulation. These energy efficiency gains are achieved while maintaining a loss rate of $1.6 \%$ which is not substantially higher than the XE1205 loss rate of $0.6 \%$ and much lower than the $43.0 \%$ loss rate of the $\mathrm{CC} 2420$ radio. These results validate our simulation study and show that substantial senderside energy gains are acheivable by opportunistically using the CC2420 radio, while providing a loss rate comparable to that of the XE1205 radio.

Figure $8(\mathrm{~b})$ shows the amount of energy consumed at the receiver as a result of the decisions made by the sender. The energy efficiency of the receiver will always fall somewhere between the efficiency of the XE1205 and CC2420 radios, depending on how often each is used. Bringing up both radio interfaces is an unavoidable result of the radio switching protocol and represents overhead beyond that of a single radio strategy. Additionally, transition times from sleep to idle/receive mode represent overhead. Our evaluation shows that the dual-radio protocol used $70 \%$ less energy than the XE1205, but $13 \%$ more energy than the CC2420. It is important to note that the receiver uses an order of magnitude less power than the sender, which means the sender-side gains overshadow the receiver-side losses.

Finally, we provide a breakdown of the percentage of packets the receiver spends in each state of the switching protocol in Table V. The receiver spends $10.7 \%$ of time in the HIGH-ON state, $78.1 \%$ of time in the LOW-ON state and $11.2 \%$ of time in the BOTH-ON state. Ideally the radio switching protocol will only force the receiver into the BOTHON state while exploring or handing off between radios. Exploration accounts for $4 \%$ of this time, while the other $7.2 \%$ is caused by explicit handoffs and timeouts.

\section{F. Microbenchmarks}

In this section, we briefly discuss measurement-based latency and energy consumption microbenchmarks based on our implementation of the unified link layer. As shown in Table VI, the energy/latency overhead imposed on the CPU by our multiradio adaptation algorithm implementation on the Arthropod is highly efficient and consumes less than a hundredth of the energy/latency of the radios used. This shows that the overhead introduced by software can be compensated by larger performance gains achieved through intelligent radio selection. The amount of memory overhead of our implementation is 111 bytes, which is a very small portion of the available
TABLE VII

DRIVER PERFORMANCE FOR MAC LAYER OPERATIONS

\begin{tabular}{|c|c|c|}
\hline & XE1205 Radio & CC2420 Radio \\
\hline \hline Ack RTT & $1.79 \mathrm{~ms}$ & $1.04 \mathrm{~ms}$ \\
\hline Ack Timeout & $2.6 \mathrm{~ms}$ & $2.4 \mathrm{~ms}$ \\
\hline Avg. Congestion Backoff & $10.4 \mathrm{~ms}$ & $10.4 \mathrm{~ms}$ \\
\hline Channel Sense Time & $1.6 \mathrm{~ms}$ & $.756 \mathrm{~ms}$ \\
\hline Sleep to Active Mode Transition Time & $1.5 \mathrm{~ms}$ & $0.58 \mathrm{~ms}$ \\
\hline
\end{tabular}

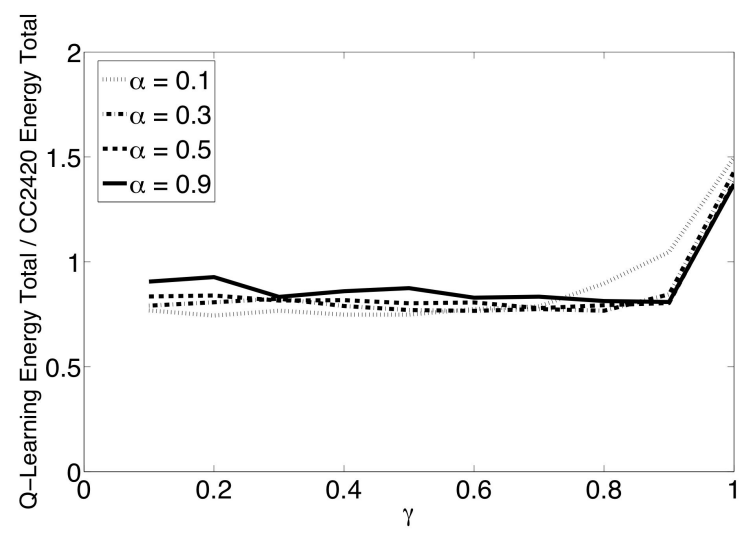

Fig. 9. Cumulative energy performance increase for different parameter values

$10 \mathrm{kB}$. A much larger portion of program memory is required, however, because two radio stacks need to be instantiated; supporting an additional radio stack requires an additional $12 \mathrm{kB}$ resulting in a total usage of $29 \mathrm{kB}$ out of the available $48 \mathrm{kB}$ of program memory, although we believe that this can be optimized considerably.

We also micro-benchmarked MAC layer operations in our TinyOS drivers. Table VII shows the individual components based on which the reward matrix is populated as described in Section IV. These measurements were used to compute the total energy cost of a transmitted packet and account for overheads in the state transitions of a receiver.

\section{G. Parameter Sensitivity}

In this section we study the sensitivity of Q-learning to its parameters: $\alpha, \gamma$ and $\epsilon$. To demonstrate the algorithm's robustness to parameter variation, we use our mobility dataset to show how the total energy consumption of the Q-Learning algorithm changes with respect to changes in parameter values. Figure 9 shows a number of plots, where for each plot we fix $\alpha$ and vary $\gamma$. The plots are very similar across several different $\alpha$ values and demonstrate that as long as $\alpha$ and $\gamma$ are chosen within a reasonable range, the performance of Q-Learning is stable. We repeated this procedure for the interference datasets, and found similar results.

Overall, we have found that a larger $\alpha$ value is generally helpful in mobility traces due to the need for fast switching. As the traces become more nomadic in nature (i.e. as they involve more waiting and less movement), the optimal choice of $\alpha$ reduces a little. However, $0.9 \leq \alpha \leq 1$ seems to be ideal in almost all settings. The choice of $\epsilon$ impacts how fast we can switch but it also impacts the energy consumption. A high $\epsilon$ can lead to more exploration overhead but is more reactive. We found that exploration roughly every 10 seconds or so provides 
a good balance but this can be tuned depending on expected dynamics. Finally, we found that the results were not very sensitive to $\gamma$, and works best in the range $0.5 \leq \gamma \leq 0.85$.

\section{CONCLUSIONS}

In conclusion, we have made three major contributions in this paper. First, we designed a new multi-radio sensor platform, the Arthropod, that pairs two radios - CC2420 and XE1205 - that offer diversity in frequency, power and range. Second, we presented the design of a Q-Learning-driven adaptive link layer that provides the abstraction of a single radio to the applications, and third, we presented a protocol that switches between radios depending on which radio offers the most energy-efficient communication channel. Experiments using a number of interference and distance datasets confirm that the system can provide effective adaptation to a range of dynamics. We also showed that the learning algorithm can be easily implemented with limited memory and computational overhead on a mote-class sensor platform.

\section{REFERENCES}

[1] Y. Agarwal, R. Chandra, A. Wolman, V. Bahl, K. Chin, and R. Gupta. Wireless wakeups revisited: Energy management for voip over wi-fi smartphones. In Proc. Mobisys, Puerto Rico, USA, June 2007.

[2] N. Banerjee, M. D. Corner, and B. N. Levine. An Energy-Efficient Architecture for DTN Throwboxes. In Proc. IEEE Infocom, May 2007.

[3] N. Banerjee, J. Sorber, M. D. Corner, S. Rollins, and D. Ganesan. Triage: A Power-Aware Software Architecture for Tiered Microservers. In Proc. Mobisys, Puerto Rico, USA, June 2007.

[4] J. Burgess, B. Gallagher, D. Jensen, and B. N. Levine. MaxProp: Routing for Vehicle-Based Disruption-Tolerant Networks. In Proc. IEEE INFOCOM, April 2006.

[5] R. Draves, J. Padhye, and B. Zill. Routing in multi-radio, multi-hop wireless mesh networks. In Proc. ACM MobiCom, pages 114-128, Philadelphia, PA, September 2004.

[6] H. Dubois-Ferrire, R. Meier, L. Fabre, and P. Metrailler. TinyNode: A Comprehensive Platform for Wireless Sensor Network Applications. In Information Processing in Sensor Networks (IPSN 2006), 2006.

[7] J. Gummeson, D. Ganesan, M. D. Corner, and P. Shenoy. An adaptive link layer for heterogeneous multi-radio mobile sensor networks. Technical Report UM-CS-2010-013, University of Massachusetts, 2010.

[8] H.-Y. Hsieh and R. Sivakumar. A transport layer approach for achieving aggregate bandwidths on multi-homed mobile hosts. In Proc. ACM Mobicom, Atlanta, GA, September 2002.

[9] P. Juang, H. Oki, Y. Wang, M. Martonosi, L. Peh, and D. Rubenstein. Energy-efficient computing for wildlife tracking: Design tradeoffs and early experiences with zebranet. In ASPLOS, San Jose, CA, October 2002.

[10] H. Jun, M. H. Ammar, M. D. Corner, and E. Zegura. Hierarchical Power Management in Disruption Tolerant Networks with Traffic-Aware Optimization. In Proc. ACM SIGCOMM Workshop on Challenged Networks (CHANTS), September 2006.

[11] D. Lymberopoulos, B. Priyantha, M. Goraczko, and F. Zhao. Towards energy efficient design of multi-radio platforms for wireless sensor networks. In Proc. of IPSN, St. Louis, MO, April 2008.

[12] N. Mishra, K. Chebrolu, B. Raman, and A. Pathak. Wake-on-WLAN. In Proc. Intl Conf on the World Wide Web (WWW), pages 761-769, 2006.

[13] V. Navda, A. P. Subramanian, K. Dhanasekaran, A. Timm-Giel, and S. Das. Mobisteer: using steerable beam directional antenna for vehicular network access. In MobiSys, New York, NY, USA, 2007.

[14] T. Pering, Y. Agarwal, R. Gupta, and R. Want. CoolSpots: Reducing the Power Consumption of Wireless Mobile Devices with Multiple Radio Interfaces. In Proc. ACM MobiSys, pages 220-232, June 2006.

[15] T. Pering, V. Raghunathan, and R. Want. Exploiting Radio Hierarchies for Power-Efficient Wireless Device Discovery and Connection Setup. In VLSI Design, pages 774-779, 2005.

[16] J. Polastre, J. Hill, and D. Culler. Versatile low power media access for wireless sensor networks. In Proc. Second ACM Conference on Embedded Networked Sensor Systems (SenSys), November 2004.
[17] A. Rahmati and L. Zhong. Context-for-wireless: Context-sensitive energy-efficient wireless data transfer. In Proc.Mobisys, Puerto Rico, USA, June 2007.

[18] J. Redi, S. Solek, K. Manning, C. Partridge, R. Rosales-Hain, R. Ramanathan, and I. Castineyra. Javelen - an ultra-low energy ad hoc wireless network. In Ad Hoc Networks, Vol. 6, No. 1, January 2008.

[19] http://www.rfsolutions.co.uk. RF Solutions: EasyBee Zigbee Transceiver.

[20] P. Rodriguez, R. Chakravorty, J. Chesterfield, I. Pratt, and S. Banerjee. Mar: A commuter router infrastructure for the mobile internet. In Proc. Second International Conference on Mobile Systems, Applications, and Services, Boston, MA, June 2004.

[21] C. Sengul, A. Harris, M. Bakht, T. Abdelzaher, and R. Kravets. Improving energy conservation using bulk transmission over high-power radios in sensor networks. In Proc. ICDCS, Beijing, China, June 2008.

[22] E. Shih, P. Bahl, and M. J. Sinclair. Wake on Wireless: An event driven energy saving strategy for battery operated devices. In Proc. ACM MobiCom, Atlanta, GA, September 2002.

[23] T. Stathopoulos, M. Lukac, D. McIntire, J. Heidemann, D. Estrin, and W. Kaiser. End-to-end routing for dual-radio sensor networks. In Proc. IEEE Infocom 2007, Anchorage, Alaska, USA, May 2007.

[24] R. S. Sutton and A. G. Barto. Reinforcement Learning: An Introduction. The MIT Press, 1998.

[25] TinyOS Website. http://www.tinyos.net/.

[26] http://www.maxstream.com. XTend OEM RF Module for Long-range Data Transfer.

[27] W. Ye, J. Heidemann, and D. Estrin. An energy-efficient mac protocol for wireless sensor networks. In Proc. IEEE INFOCOM, pages 15671576, New York, NY, USA, June 2002.

[28] J. Zhu, A. Waltho, X. Yang, and X. Guo. Multi-radio coexistence: Challenges and opportunities. In Proc. ICCCN, August 2007.

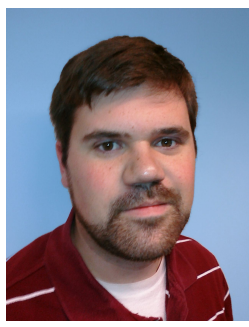

Jeremy Gummeson Jeremy Gummeson is currently a PhD student in the Department of Electrical Engineering at the University of Massachusetts Amherst. $\mathrm{He}$ received his B.S and M.S. Degrees from the University of Massachusetts Amherst in 2006 and 2010, respectively. His research interests are in Sensor Networks, Energy Harvesting, and RFID.

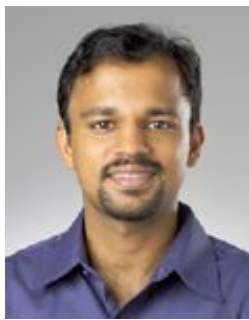

Deepak Ganesan Deepak Ganesan is currently Assistant Professor in the Department of Computer Science at UMASS Amherst. He received his Ph.D. in Computer Science from UCLA in 2004 and his bachelors in Computer Science from IIT, Madras in 1998. He received the NSF CAREER Award in 2006 and the IBM Faculty Award in 2008. He was selected as a UMass Junior Faculty Fellow in 2008, and a UMass Lilly Teaching Fellow in 2009. He is a Program co-chair for ACM SenSys 2010.

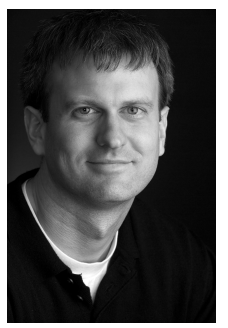

Mark Corner Mark Corner has been an Associate Professor in the Department of Computer Science at the University of Massachusetts Amherst since 2003. He graduated with his $\mathrm{PhD}$ in Electrical Engineering from the University of Michigan. His interests are in mobile and pervasive computing and networking, file systems, and security. He received an NSF CAREER award in 2005, Best Paper Awards at FAST 2007 and ACM Multimedia 2005, and the Best Student Paper Award at Mobicom 2002. Prof. Corner serves on the editorial board of IEEE 


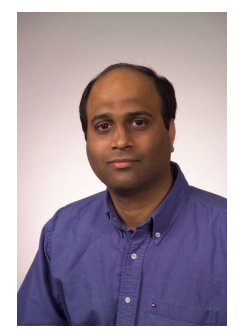

Prashant Shenoy Prashant Shenoy received the B.Tech degree in Computer Science and Engineering from the Indian Institute of Technology, Bombay in 1993, and the M.S and Ph.D degrees in Computer Science from the University of Texas, Austin, in 1994 and 1998, respectively. He is currently a Professor of Computer Science at the University of Massachusetts Amherst.

His research interests are in operating and dis- tributed systems, sensor networks, Internet systems and pervasive multimedia. He has been the recipient of the National Science Foundation Career Award, the IBM Faculty Development Award, the Lilly Foundation Teaching Fellowship, the UT Computer Science Best Dissertation Award, an IIT Silver Medal, and best paper awards at USENIX, ACM Multimedia and IEEE WISE. He is a senior member of the IEEE and a distinguished member of the ACM. 